\title{
Efecto inhibitorio in vitro de cinco isotiocianatos sobre Rhizoctonia solani Kühn AG-3
}

\author{
Luis Federico Molina-Vargas ${ }^{1} \&$ José Ulloa Bentura-Castellanos ${ }^{2}$ \\ 111fmolinav@yahoo.com \\ ${ }^{1}$ Buenas Prácticas Agrícolas Ltda (BPA Ltda) \\ Dirección: Calle 35B No 16c-52, Tunja- Colombia \\ ${ }^{2}$ Universidad Nacional Abierta y a Distancia -UNAD
}

\begin{abstract}
Resumen.- Evaluamos el efecto de cinco isotiocianatos (ITC) para controlar a Rhizoctonia solani Kühn AG-3, un hongo causante de enfermedades en plantas de importancia agrícola, como estudio preliminar para la implementación de la técnica de biofumigación en Colombia. Realizamos pruebas in vitro con diluciones de cinco ITC, con metanol como solvente, en cajas de Petri con medio PDA y calculamos las áreas de crecimiento micelial, el porcentaje de inhibición y la concentración efectiva media (CE50). La respuesta del patógeno fue dependiente de la concentración y del tipo de ITC aplicado. Todos los ITC en concentraciones al 10\% presentaron efecto inhibitorio total. PEITC y BITC tuvieron un efecto fungistático hasta los seis y 27 días después de aplicados los tratamientos, respectivamente. PITC, AITC y MITC mantuvieron el efecto inhibitorio por más de un año después de aplicados los tratamientos. PITC fue más eficaz en controlar R. solani Kühn AG-3 que los otros ITC, mostrando el valor más bajo de la CE50 para la inhibición del crecimiento micelial.
\end{abstract}

Palabras clave: biocontrol, biofumigación, Brassicaceae, fungistático, glucosinolato, isotiocianatos.

\begin{abstract}
We tested the potential use of five isothiocyanates (ITC) to control Rhizoctonia solani Kühn AG-3, the fungus that produces several plant diseases. This is a preliminary study in order to apply biofumigation technique in Colombia,. We used dilutions of five ITC with methanol as a solvent, which were applied in Petri dishes with PDA medium. We calculated mycelium growth areas, inhibition percentage, and 50 effective concentrations (EC50) through digital pictures we took 72 hours after treatments were applied. The answer of the pathogen depended on concentration and type of ITC. All ITC in 10\% concentration presented total inhibitory effect. PEITC and BITC have a fungistatic effect until six and 27 days after treatments were applied, respectively. PITC, AITC, and MITC kept inhibitory effect more than one year after treatments were applied. PITC was more efficient in controlling $R$. solani Kühn AG-3 than the other ITC, with the lowest value of EC50 for inhibition of mycelial growth.
\end{abstract}

Key words: biocontrol, biofumigation, Brassicaceae, fungistatic, glucosinolate, isothiocyanates.

\section{Introducción}

Los cultivos de plantas de la familia Brassicaceae (crucíferas), usados en rotaciones de cultivos como abonos verdes, han sido asociados con reducciones de patógenos y plagas del suelo. Estas reducciones han sido atribuidas a la producción de componentes de azufre volátiles en un proceso conocido como biofumigación (Larkin \& Griffin 2007). Las sustancias naturales con actividad biocida como los aceites esenciales (Tsao \& Zhou 2000), los compuestos aromáticos (Neri et al. 2005) y las moléculas producidas por varios sistemas de defensa de las plantas, están siendo estudiados por su marcada acción fungicida. Este último grupo de componentes incluye algunas moléculas como los isotiocianatos (ITC), provenientes de la hidrólisis enzimática de los glucosinolatos (GSL), que en pruebas biológicas específicas han mostrado una fuerte actividad contra un amplio rango de patógenos 
con prometedores resultados en diferentes situaciones (Mari et al. 2008). Se han aislado y caracterizado aproximadamente 120 GSL de especies de plantas de la familia Brassicaceae y familias relacionadas (Wittstock \& Halkier 2002), como Capparidaceae, Moringaceae, Resedaceae, Stegnospermaceae y Tovariaceae $y$ varias familias no relacionadas como Caricaceae, Limnanthaceae y Tropaeolaceae (Vaughn \& Berhow 2005). Los GSL están almacenados en vacuolas y son liberados al sufrir daño celular, luego de lo cual entran en contacto con la enzima mirosinasa (tioglucosidasa encontrada en plantas que contienen GSL) y son hidrolizados en diferentes productos (Fimognari et al. 2002, Zhang et al., 2005), incluyendo los ITC, nitrilos, tiocianatos, epitionitrilos y oxazolidinetionas, que varían dependiendo de la especie vegetal, la sustitución de la cadena, el $\mathrm{pH}$ de la célula y la concentración de hierro (Vaughn \& Berhow 2005).

Algunas enfermedades presentes en cultivos de importancia agrícola, como el "damping-off" o marchitamiento fúngico, la podredumbre de la raíz, la mustia hilachosa, el cáncer del tallo, la pudrición de la corona, yemas y frutos, y la quemazón del follaje, son causados por la presencia del hongo fitopatógeno Rhizoctonia solani Kühn (Blazier \& Conway 2004).El amplio rango de perfiles de GSL y la diferencia de toxicidad de los ITC involucrados contra las diferentes plagas vegetales, ofrecela posibilidad de seleccionar especies de plantas Brassicaceae con mayor potencial de biofumigación en los organismos de interés (Kirkegaard et al. 1998). Realizamos experimentos in vitro para evaluar la eficacia de cinco ITC contra $R$. solani Kühn y proponer el uso posterior de plantas de la familia Brassicaeae para controlar el patógeno mediante la técnica de la biofumigación, como alternativa a la prohibición del uso de algunos fumigantes sintéticos.

\section{Materiales y métodos}

Mediante un experimento in vitro evaluamos el efecto de cinco ITC sobre $R$. solani Kühn AG-3 (del inglés, anastomosis group three: grupo de anastomosis tres), obtenido de la colección del laboratorio de Biotecnología de la Facultad de Agronomía de la Universidad Nacional de Colombia, sede Bogotá. Preparamos el inóculo en una cámara de flujo laminar, sembrando micelio de $R$. solani Kühn
AG-3 en el centro de cajas de Petri con medio PDA al 3.9\%. Al cabo de cinco días cortamos discos de $5 \mathrm{~mm}$ de los bordes de las colonias y los transferimos a cajas de Petri con medio PDA.

Evaluamos cada ITC independientemente mediante experimentos separados, con un diseño experimental de bloques completamente aleatorizado (DBCA), en donde los bloques correspondieron a tres repeticiones del experimento bajo las mismas condiciones. Las unidades experimentales fueron cajas de Petri con medio PDA al $3.9 \%$ y discos de micelio de $R$. solani Kühn AG-3 de cinco días de sembrado. Utilizamos cinco réplicas por tratamiento, siendo los tratamientos las concentraciones de cinco ITC al $0.0 \%, 0.01 \%, 0.1 \% 1.0 \%$ y $10.0 \%$, utilizando metanol como solvente en una relación de aguametanol de 80:20. Utilizamos allyl ITC al 93\% (AITC), benzyl ITC al 98\% (BITC), phenyl ITC al 93\% (PITC) y phenethyl ITC al 99\% (PEITC) obtenidos de Sigma Aldrich y methyl ITC al 98\% (MITC) obtenido del producto Dazomet (Basamid ${ }^{\circledR}$-BASF) como testigo comercial. Una vez aplicados los tratamientos sellamos las cajas de Petri con doble capa de papel parafilm y las mantuvimos en cámara de extracción de gases a temperatura ambiente. Pasadas 72 horas desde la aplicación de los tratamientos (72 hdt) tomamos fotografías digitales de las unidades experimentales y estimamos las áreas de crecimiento micelial con el software MapMaker ${ }^{\circledast}$ versión 3.5. Calculamos el porcentaje de inhibición a las $72 \mathrm{hdt}$ con la fórmula propuesta por Taylor et al. (2002), restando el área del inóculo inicial. Para los análisis estadísticos utilizamos los procedimientos Means, Univariate, Glm y Reg del paquete estadístico SAS (Statistical Analysis System) versión 9.0. Para obtener los valores de la concentración efectiva media (CE50) correspondiente a la concentración de ITC que inhibe el crecimiento del micelio en un $50 \%$, reemplazamos el valor " 50 " en las ecuaciones resultantes que relacionan la variable respuesta, el porcentaje de inhibición (y), con la concentración de ITC (x).

\section{Resultados}

Todos los ITC evaluados presentaron efecto inhibitorio dependiente de la concentración. En concentraciones máximas del 10\%, todos los ITC tuvieron un efecto inhibitorio 


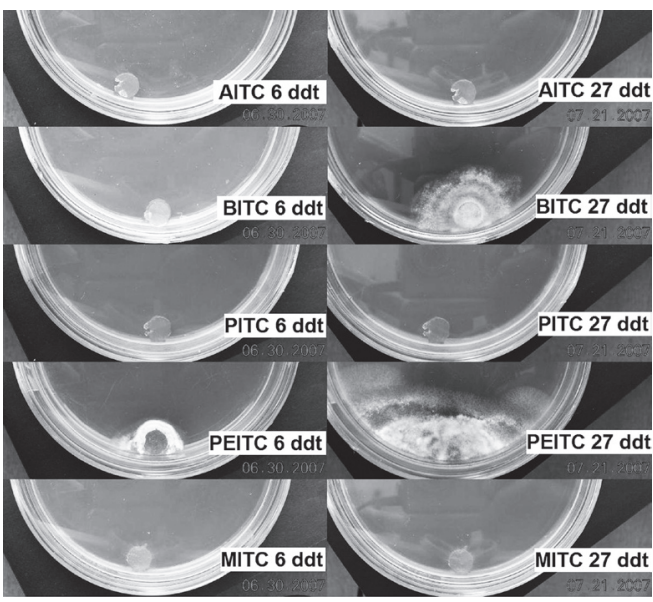

Figura 1. Efecto inhibitorio de los isotiocianatos al 10\%. La inhibición del crecimiento micelial in vitro de cinco ITC contra R. solani Kühn AG-3 se evaluó a los seis y a los 27 días después de aplicados los tratamientos (ddt).

total en $R$. solani Kühn AG-3 (Figura 1), exceptuando PEITC a los seis días después de aplicados los tratamientos (ddt). Por otra parte, los tratamientos con BITC mostraron crecimiento del hongo después de $27 \mathrm{ddt}$, lo que sugiere un biocontrol por debajo de este rango de tiempo.AITC, PITC y MITC (testigo comercial) mostraron un efecto fungistático más prolongado, que se mantiene incluso un año después de iniciados los tratamientos. Con base en la CE50 (Figura 2) la sensibilidad de $R$. solani Kühn AG-3 fue mayor para PITC y AITC, que para el testigo comercial MITC.

\section{Discusión y Conclusiones}

Nuestros resultados confirman los reportes sobre la alta toxicidad de AITC contra $R$. solani (Sarwar et al. 1998, Chung et al. 2002, Dhingra et al. 2004). Observamos completa inhibición en concentraciones superiores a $1.000 \mathrm{ppm}$, valores más altos que los reportados por Chung et al. (2002) y Dhingra et al. (2004) quienes encontraron completa inhibición con AITC por encima de 500 ppm y $50 \mathrm{ppm}$, respectivamente. Sin embargo ellos no reportan la CE50, la cual difiere dependiendo del momento de evaluación y tipo de aislamiento (Molina 2008). Sarwar et al. (1998) encontraron que $R$. solani era

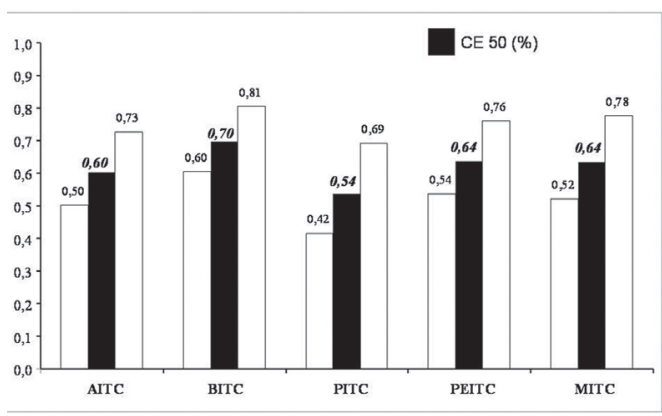

Figura 2. Concentración efectiva media. Valores de la CE50 para todos los ITC 72 horas después de aplicados los tratamientos. Mediante las barras blancas se representan los límites inferiores y superiores del intervalo de confianza.

sensible in vitro a MITC y BITC, entre otros ITC, reduciendo el crecimiento micelial, siendo los ITC aromáticos menos tóxicos debido a su volatilidad.

El experimento mostró la ventaja del uso de los ITC como biocontroladores de $R$. solani Kühn AG-3 en comparación con el fumigante sintético basado en MITC (Dazomet: Basamid $^{\oplus}$ ), lo que confirma los resultados de Kirkegaard et al. (1996) y Sarwar et al. (1998) quienes trabajaron con ITC liberados de GSL provenientes de tejidos de plantas Brassicaceae. Por otra parte, Larkin \& Griffin (2007) redujeron los niveles de inóculo de $R$. solani entre un 20 y 56\%, mediante pruebas en invernadero con cultivos de brasicáceas y cebada. Una de las principales limitaciones en nuestro experimento fue el uso de un solo aislamiento pues Kirkegaard et al. (1996) encontraron variación del efecto de AITC en diferentes aislamientos de $R$. solani, en un rango entre 10 al $60 \%$ de los tratamientos control, mediante la liberación de altos contenidos de AITC (2-propenyl ITC) utilizando tejidos de plantas Brassicaceae. Proponemos en estudios futuros, evaluar los ITC en otros aislamientos de $R$. solani. Estos resultados indican que las plantas de la familia Brassicaceae con altos contenidos de GSL tienen un potencial para su uso como abonos verdes y para el control de múltiples problemas de enfermedades del suelo (Larkin \& Griffin 2007). Los abonos verdes con altos contenidos de GSL, que producen un biofumigante natural después 
de su incorporación en el suelo, son una alternativa a los productos sintéticos, con los consecuentes beneficios ambientales. Sin embargo, es posible que el amplio espectro de toxicidad de los mismos, haga daño a los organismos benéficos del suelo como los agentes de control biológico $\mathrm{u}$ otros antagonistas de plagas (Henderson et al. 2009).

\section{Agradecimientos.- Agradecemos al} laboratorio de Biotecnología de la Facultad de Agronomía de la Universidad Nacional de Colombia, sede Bogotá y a Buenas Prácticas Agrícolas Ltda (www.bpaltda.org) por la cofinanciación de este proyecto.

\section{Referencias}

Blazier, S, \& KE Conway. 2004. Characterization of Rhizoctonia solani isolates associated with patch diseases on turfgrass. Proc. Okla. Acad. Sci., 84: 41-51

Chung, WC, JW Huang, HC Huang \& JF Jen. 2002. Effect of ground Brassica seed meal on control of Rhizoctonia damping-off of cabbage. Can. J. Plant Pathol. 24: 211-218

Dhingra, OD, ML Costa \& GJ Silva Jr. 2004. Potential of allyl isothiocyanate to control Rhizoctonia solani seedling damping off and seedling blight in transplant production. Journal of Phytopathology 52: 352-357

Fimognari, C, M Nüsse, C Rossano, I Renato, G. Cantelli-Forti \& P Hrelia. 2002. Growth inhibition, cell-cycle arrest and apoptosis in human T-cell leukemia by the isothiocyanate sulforaphane. Carcinogenesis 23: 581-586

Henderson, DR, E Riga, RA Ramirez, J Wilson \& WE Snyder. 2009. Mustard biofumigation disrupts biological control by Steinernema spp. nematodes in the soil. Biological Control 48: 316-322

Kirkegaard, JA, PT Wong \& JM Desmarchelier. 1996. In vitro suppression of fungal root pathogens of cereals by Brassica tissues. Plant pathology 45: 593-603

Kirkegaard JA, M Sarwar \& JN Matthiessen.
1998. Assessing the biofumigation potential of crucifers. Acta Hort., 459: 105-111

Larkin, RP \& TS Griffin. 2007. Control of soilborne potato diseases using Brassica green manures. Crop Protection 26: 1067-1077

Mari, M, O Leoni, R Bernardi, F Neri \& S Palmieri. 2008. Control of brown rot on stonefruit by synthetic and glucosinolate-derived isothiocyanates. Postharvest Biology and Technology 47: 61-67

Molina Vargas, LF. 2008. Método in vitro para evaluar la eficacia de los isotiocianatos contra Rhizoctonia solani, mediante la determinación de la concentración efectiva media (CE50) calculando el área de crecimiento micelial con el software Mapmaker 3.5 Tesis de maestría. Facultad de Agronomía de la Universidad Nacional de Colombia. Bogotá D.C.

Neri, F, M Mari \& S Brigati. 2005. Control of Penicillium expansum by plant volatile compounds. Plant Pathol. 55: 100-105

Sarwar, M, JA Kirkegaard, PW Wong \& JM Desmarchelier. 1998. Biofumigation potential of brassicas. Plant and Soil. 201: 103-112

Taylor, RJ, B Salas, GA Secor, V Rivera \& NC Gudmestad.2002.SensitivityofNorthAmerican Isolates of Phytophthora erythroseptica and Pythium ultimum to Mefenoxam (Metalaxyl). Plant Disease 86: 797-802

Tsao, R \& T Zhou. 2000. Antifungal activity of monoterpenoids against postharvest pathogens Botrytis cinerea and Monilinia fructicola. J. Essential Oils 12: 113-121

Vaughn, SF \& MA Berhow. 2005. Glucosinolate hydrolysis products from various plant sources: $\mathrm{pH}$ effects, isolation, and purification. Industrial Crops and Products 21: 193-202

Wittstock, U \& BA Halkier. 2002. Glucosinolate research in the Arabidopsis era. Trends in Plant Science 7: 263-270

Zhang, Y, L Jun \& L Tang. 2005. Cancer-preventive isothiocyanates: dichotomous modulators of oxidative stress. Free Radical Biology \& Medicine 38: 70-77 